\title{
Sudden Death of Entanglement of Two Quantum Dots Embedded in Its Own Cavity
}

\author{
S. Sánchez-Sánchez ${ }^{1}$ J. J. Sánchez Mondragón , F. R. Castillo Soria², \\ V. I. Moreno Oliva ${ }^{1}$, E. Román Hernández ${ }^{1}$ \\ ${ }^{1}$ Instituto de estudios de la Energía, Universidad del Istmo, Carrera de Matemáticas Aplicadas, \\ Oaxaca, Mexico \\ ${ }^{2}$ Universidad del Istmo, Carrera de Ingeniería en Computación, \\ Oaxaca Mexico \\ ${ }^{3}$ Instituto Nacional de Astrofísica Óptica y Electrónica, Departamento de Óptica, \\ Puebla, Mexico \\ ssanys1@hotmail.com, ssanchez@bianni.unistmo.edu.mx,jjsanchez@inaoep.mx
}

\begin{abstract}
Entanglement of quantum systems is a key aspect to understanding the dynamics and behavior of mixed systems (density matrix) as bipartite quantum bits (qubits). Thus we need to have a reliable and accurate way to measure the entanglement degree of the system, i.e., how it evolves, so that raises several approaches to meet these demands. A quantifiable measure widely used is the "entanglement of formation" of a mixed state, defined as the minimum number required of "singlets" to create a set of pure states that represents the density matrix of the system. In this paper we consider a system of two semiconductor quantum dots embedded in its own cavity and coupled to the internal mode field of cavity type Jaynes-Cummings. The entanglement between the two quantum dots is investigated, and we show analytically that entanglement has very interesting, effects such as time evolution including the effect called sudden death.
\end{abstract}

Keywords: entanglement, quantum dots, semiconductors, sudden death.

\section{Introduction}

By several years, many authors have studied entanglement because their enormous importance at a fundamental level and because its applications to quantum information and quantum computing (Nielsen and Chuang, 2000). Entanglement has marked a new way to reinterpret the quantum nature of computer technology due to the incorporation of quantum processing units with so-called quantum bits (q-bits), represented as dual units that open up infinite possibilities of parallel processing, at least theoretically, much faster than any classical computational process.

However this has been the case at the theoretical level, therefore it is essential to implement physical models that allow the incorporation of this development into 
feasible systems or where the technological inertia may lead to, and one of the most visible are Quantum Dots (QDs). In spite that we usually refer to QDs as Atom like structures, there are substantial differences such as the exchange interaction (Förster interaction), (Sanchez-Mondragon, Alejo-Molina, S. Sanchez-Sanchez, 2005 \& 2009) which has been used as the basis for proposals of quantum computation, and therefore deserve a careful analysis.

Quantum entanglement has played very important roles in quantum information processing such as quantum teleportation (Nielsen and Chuang, 2000) quantum cryptographic, quantum dense coding (Bennett, Wiesner, 1992) and parallel computing (DiVincenzo, 1995). Therefore a precise measurement is needed to quantify the degree of entanglement for those qubits system in collaboration or competition with such exchange interaction. This is more interesting because the physical character and mathematical structure of entangled states have not been well understood and the Förster interaction tuning opens new possibilities to deal with its fundamental questions. There are two important problems for entanglement. One is to find a method to determine whether a given state is separable (or not entangled), and the other one, it is to define the best measurement quantifying an amount of entanglement of a given state. In order to solve the first problem, much effort has been made, [6-8]. The quest for proper measurement of entanglement has received also a great deal of attention. The entanglement of formation, distillation, and relative entropy, (Bennett, DiVincenzo, Smolin, Wootters, 1996) negativity, concurrence (Hill, Wootters, 1997; Wootters, 1998) concurrence related measures, or positive operator are used to investigate entanglement.

Although the entanglement of formation is defined for arbitrary-dimensional bipartite systems, so far no explicit analytic formulates for entanglement of formation have been found for systems larger than a pair of qubits, except for some special symmetric states (Terhal, Gerd, Vollbrecht, 2000).

Another serious problem that must be considered in entanglement, as mentioned earlier, in a quantum system is it may deteriorate due to interaction with background noise or with other systems usually called environments. Interest was originally concerned with the consequences for quantum measurement and the quantum-classical transition (Joos, Zeh, et. al. 2003). More recently, entanglement decoherence has been studied in connection with obstacles to realize various quantum information processing schemes. T. Yu and Eberly have shown that entanglement can decay to zero abruptly, in a finite time, a phenomenon termed entanglement sudden death (Yu and Eberly, 2004; Yu and Eberly, 2006).

Such quantum correlations are responsible for much of the challenge in understanding interacting many-body quantum systems, and it is therefore of fundamental importance to have quantitative knowledge of these correlations. Progress in quantum information theory has led to the development of new measures of the inseparability of a quantum state, and in the last few years these measures have been used to assess the quantum correlations in diverse physical systems. Concurrence is an especially useful metric for such studies because it can be applied to mixed as well as pure states. It therefore can be used to quantify the thermal entanglement in a system at nonzero temperature. It can also be applied to evaluate the inseparabililty of an equal incoherent mixture of degenerate energy 
eigenstates. However as we mentioned above, concurrence is defined only for a pair of qubits. Since a qubit is formally equivalent to a spin-1/2 particle when only the spin degree of freedom of the latter is considered, this has led to several analyses of the thermal entanglement between a pair of interacting spin-1/2 particles. Entanglement of formation, is another important entanglement measure, which can be calculated directly from the concurrence and is monotonically related to it. The method of calculating the concurrence for more general density matrices can be found in Wootters.

The importance of this issue is to find necessary and sufficient conditions for the development of quantum computer systems in their physical implementation (hardware) and the new rules of quantum processing (software). In our case we focus on studying the physical implementation on a fundamental level, seeking the most appropriate quantum physical system of many systems studied in quantum physics for many decades to more sophisticated atomic systems with cooperative and collective effects. Up until now, such quantum-mechanical computers have been proposed in terms of trapped ions and atoms, cavity quantum electrodynamics (QED), nuclear magnetic resonance, Josephson junctions, and semiconductor nanostructures schemes. However all of the above proposals have decoherence and operational errors as the main obstacles for their experimental realization, which pose much stronger problems here than in classical computers. There is much current excitement about the possibility of using solid-state-based devices for the reliable rendering of quantum computation tasks.

In particular, semiconductor nanostructure fabrication technology is well developed and hence offers us a wide and promising arena for the challenging project of building quantum information processors. Because of their quantummechanical nature and their potential scalability properties, semiconductor quantum dots (QDs) are very promising candidates for the implementation of quantum computing processes. Several solid-state design schemes for quantum computation have been proposed to date: Kane (B.E. Kane, 1998) has proposed a scheme that encodes information onto the nuclear spins of donor atoms in doped silicon electronic devices where externally applied electric fields are used to perform logical operations on individual spins. Loss and Di-Vincenzo have presented a scheme based on electron spin effects, in which coupled quantum dots are used as a quantum gate. This scheme is based on the fact that the electron spins on the dots have an exchange interaction (Forster interaction) which changes sign with increasing external magnetic field.

In this paper we consider a double quantum dot system coupled to the mode Jaynes-Cummings cavity type, thus we investigate the entanglement between two quantum dots, each embedded in his own cavity, and we show analytically that entanglement has interesting effects such as temporal evolution, as well as the socalled sudden death effect. The system composed of two quantum dots were previously entangled before that these were immersed into the cavity. We study this system in the context of cavity quantum electrodynamics (C-QED). However we must to clarify that this is not a paper on QDs systems for quantum computing applications. It's just a proposal about semiconductors QDs in quantum optics and C-QED fundamentals, which we used to do the theoretical study, also supported by 
the entanglement of formation procedure as a quantitative measure for the entanglement between our qubits: i.e. the two quantum dots system more the cavities.

\section{Concurrence and Entanglement of Formation}

Entanglement is a fundamental insight of quantum mechanics, which corresponds to the presence of nonlocal correlations between different parts of a system that cannot be explained classically. That is, a pure state of a pair of quantum systems (bipartite) is called entangled if it is not factorizable (i.e., if the state total cannot be written as a product of states of the particle) and a mixed state is entangled if it can be represented as the mixed state pure factorizable. For both pure and mixed quantum states, there are good measures of the degree of entanglement. In the case of pure states of a bipartite system there is a single widely accepted measure of entanglement, whereas for mixed states of such systems there are three measures that have been extensively studied. One of these, entanglement of formation, is a subject of this paper. We use the concurrence of Woosters as a measure in this work, mainly for its importance for mixed states and the convenience of its definition and normalization.

\subsection{Entanglement of Formation for a System of Two QDs}

The key element in the quantum information processing is the so-called quantum bit. For this reason, understanding their behavior in quantum computing environments is essential to carry out external operations that perform specific calculations in locations on qubits by logic operations with new algorithms adapted to these qubits. So we should form networks of qubits at different intervals making full operations. In our case we have a small network of two QDs at the nodes of network under this study we will provide the means to insight the transfer at a distance of entanglement in the lattice network.

Our qubits are a system of two quantum dots which are located in their respective single-mode ( $a, a^{\dagger}$ and $b, b^{\dagger}$ ), lossless cavities so that a cavity includes only one such dots. Thus, each node of our network consists of a cavity in which there is a QD. We will restrict our attention to the dynamics of entanglement between two such nodes. We will denote the dot at the first node by $A$, cavity at the first node by $a$, dot at the second node by $B$ and cavity at the second node by $b$, as sketched in Fig. 1 . We are going to be using the QDs Hamiltonian model (Sánchez-Sánchez, 2011).

Quiroga-Jhonson 1999; Reina-Quiroga-Jhonson ) to specify the interactions in our system, this include the Förster interaction. The QDs Hamiltonians (defined the constant $\hbar=1$ ) as we will use in next sections, i.e. equations (1), (2) and (3). We consider $L$ identical semiconductor quantum dots that are equally coupled to each other via coulombic interaction. The QDs interact with a quantized field (dipole interaction) in a high-Q cavity. Then the coupled QD-field system is described by the Hamiltonian (Sanchez-Mondragon, Alejo-Molina, S. Sanchez-Sanchez, 2005 \& 2009. Quiroga and Johnson 1999). 


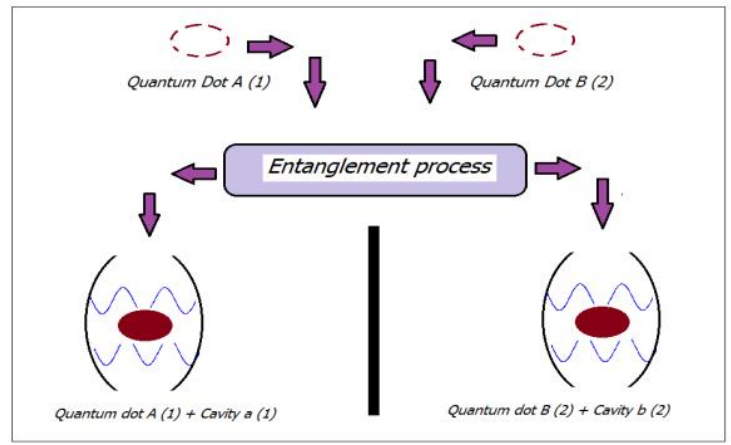

Fig. 1. This diagram show our system of two QDs previously entangled. The QDs are placed in their respective cavity, which there are not interaction between them.

With respect to two subsystems A and B

$$
\begin{aligned}
& H^{(A)}=\omega a^{\dagger} a+\varepsilon J_{z}^{a}+g_{a}\left(J_{+}^{a} a+a^{\dagger} J_{-}^{a}\right)+W_{a}\left(J_{a}^{2}-\left(J_{z}^{a}\right)^{2}\right), \\
& H^{(B)}=\omega b^{\dagger} b+\varepsilon J_{z}^{b}+g_{b}\left(J_{+}^{b} b+b^{\dagger} J_{-}^{b}\right)+W_{b}\left(J_{b}^{2}-\left(J_{z}^{b}\right)^{2}\right),
\end{aligned}
$$

where $\varepsilon$ is the QD band gap, $g_{a}=g_{b}=g$ is the coupling strength between the field and the QDs, $\omega$ is the field frequency, and $W_{a}=W_{b}=W$, represents the interdot coulomb interaction.

The coulomb interaction process known as Forster process exchanges energy, but does not require the physical transfer of the electrons and holes. For equal coupling these QDs are equidistant from each other so that the dots lie on a line for cases $L=2$, at the vertices of an equilateral triangle for $L=3$, and at the vertices of a regular pyramid for $L=4$. The Hamiltonian (1) can be rewritten in a much more suitable in the representation of angular momentum, with the changes point out into references: (Sanchez-Sanchez S. 2011; Sanchez-Mondragon, Alejo-Molina, Sanchez-Sanchez S., 2005 \& 2009. Quiroga and Johnson 1999), we obtained that may consist of two parts, first one with the Dicke $H_{D k}$ Hamiltonian itself and the other one is the interaction Hamiltonian Förster $H_{F}$, defined as $H_{D k_{A . B}}=\Delta J_{z}^{a, b}+g\left(J_{+}^{a, b} a+a^{\dagger} J_{-}^{a, b}\right)$ and $H_{F}=W\left(J_{a, b}^{2}-\left(J_{z}^{a, b}\right)^{2}\right)=W J_{+}^{a, b} J_{-}^{a, b}$ where the constant $\Delta=\varepsilon-\omega$ is the detuning between the electromagnetic field and the band-gap.

The Hamiltonian of $L$ QDs can be rewritten in the form: $H=\omega N+Q_{L}$, with $N=a^{\dagger} a+J_{z}+L / 2$ is the number of atoms and photons and $Q_{L}=H_{D k}+H_{F}$ are constants of motion. It should be noted that the term of Förster $W J_{+}^{a, b} J_{-}^{a, b}$ is nonlinear. Furthermore we introduced a new constants $\varepsilon^{\prime}$, defined as $\varepsilon^{\prime}=\varepsilon-W$. However there is another way to rewrite the Hamiltonian (1), using the relations of 
the algebra of angular momentum, thus we rewrite the Hamiltonian to include explicitly the detuning $\Delta$ which now call Förster Detuning $\Delta^{\prime}$, so we obtain:

$$
\begin{aligned}
& H^{(A)}=\omega\left(a^{\dagger} a+J_{z}^{a}\right)+g\left(J_{+}^{a} a+a^{\dagger} J_{-}^{a}\right)-\Delta^{\prime} J_{z}^{a}+W J_{+}^{a} J_{-}^{a}, \\
& H^{(B)}=\omega\left(b^{\dagger} b+J_{z}^{b}\right)+g\left(J_{+}^{b} b+b^{\dagger} J_{-}^{b}\right)-\Delta^{\prime} J_{z}^{b}+W J_{+}^{b} J_{-}^{b},
\end{aligned}
$$

while the parameters are defined as: $\Delta^{\prime}=\Delta+\mathrm{W}=\omega-\varepsilon+W=\omega-\varepsilon^{\prime}$. In a frame rotating with the field frequency $\omega$, Eq. (2) takes the form:

$$
\begin{aligned}
& H_{F}^{(A)}=\Delta^{\prime} J_{z}^{a}+g\left(J_{+}^{a} a+a^{\dagger} J_{-}^{a}\right)+W J_{+}^{a} J_{-}^{a}, \\
& H_{F}^{(B)}=\Delta^{\prime} J_{z}^{b}+g\left(J_{+}^{b} b+b^{\dagger} J_{-}^{b}\right)+W J_{+}^{b} J_{-}^{b} .
\end{aligned}
$$

\section{Two QDs Interacting with their Own Quantized Cavity Field: Hamiltonian Diagonalization}

From now on we will use this Hamiltonian (3), for each of the subsystems in order to diagonalizar the Hamiltonian, i.e. splits into two subsystems which are represented as $H_{T}=H^{(A)}+H^{(B)}$. This will simplify the task of studying the time evolution of the QD-field system. Starting with the initial condition representing the vacuum of excitons (S. Sánchez-Sánchez, 2011) (Sanchez-Mondragon, Alejo-Molina, S. Sanchez-Sanchez, 2005 \& 2009. Quiroga and Johnson 1999. And Mitra, Vyas, Erenso, 2007), $|j=1 / 2, m=-1 / 2\rangle=|\downarrow\rangle \sqrt{a^{2}+b^{2}}$, only the $j=1 / 2$ subspace is optically active while the $j=0$ subspace remains dark.

We choose the basis of eigenstates of $J^{2}$ and $J_{z},|\downarrow\rangle=|j=1 / 2, m=-1 / 2\rangle$, $|\uparrow\rangle=|j=1 / 2, m=1 / 2\rangle$, as an appropriate representation for this problem represents the vacuum for excitons, $|\uparrow\rangle$ denotes a symmetric delocalized singleexciton state. If we represent the field state intro each cavity by the Fock state $\left|n_{a}, n_{b}\right\rangle$ and consider the QDs in the entangled state involving the vacuum and exciton states $[|\uparrow \downarrow\rangle \pm|\downarrow \uparrow\rangle]$, then we will have an invariant subspace spanned by the tensor product $\{|\uparrow \downarrow\rangle \otimes|00\rangle ;|\downarrow \uparrow\rangle \otimes|00\rangle ;|\downarrow \downarrow\rangle \otimes|10\rangle ;|\downarrow \downarrow\rangle \otimes|01\rangle\}$.

With these basis vectors we determine the matrix elements of the Hamiltonian in Eq. (3) and obtain the eigenvalues, and the eigenvectors by mean of diagonalization. The explicit matrix is

$$
H_{T}=H^{(A)}+H^{(B)}=\left(\begin{array}{cccc}
W & 0 & g & 0 \\
0 & W & 0 & g \\
g & 0 & \Delta^{\prime} & 0 \\
0 & g & 0 & \Delta^{\prime}
\end{array}\right),
$$


where $\Delta^{\prime}=\omega-\varepsilon^{\prime}=\Delta+W$. An interesting case is for $\Delta^{\prime}=\omega-\varepsilon^{\prime}=\Delta+W=0+W=W$, that is, when we have resonance. In next subsection we will use this case in order to calculate the Concurrence function and thus the Entanglement of Formation, for now we calculate the general case for the Hamiltonian diagonalization. The characteristic polynomial for matrix (4) is given by $P(\lambda)=\left[g^{2}+(W-\lambda) \lambda-(W-\lambda) \Delta^{\prime}\right]^{2}$.

At both cavities with the same field frequency $\omega$, and we define the constants for simplicity as $\delta=\left[4 g^{2}+W^{2}-2 W \Delta^{\prime}+\left(\Delta^{\prime}\right)^{2}\right]^{1 / 2}=\left[W\left(W-2 \Delta^{\prime}\right)+\left(4 g^{2}+\left(\Delta^{\prime}\right)^{2}\right)\right]^{1 / 2}$ the eigenvalues take the form: $\lambda_{E 1, E 2}=(1 / 2)\left(W+\Delta^{\prime}-\delta\right), \quad \lambda_{E 3, E 4}=(1 / 2)\left(W+\Delta^{\prime}+\delta\right)$; Due to the tensor product of the quantum states $|j, k\rangle=|j\rangle \otimes|k\rangle$ form a four-dimensional basis in the Hilbert space $\mathrm{SU}(2) \otimes \mathrm{SU}(2)$. And the corresponding normalized eigenvectors are:

$$
\begin{aligned}
& \left|\lambda_{1}\right\rangle=\left(4 g^{2}+\Omega_{1}^{2}\right)^{-1 / 2}\left[\left(\Omega_{1}|\uparrow \downarrow, 00\rangle+2 g|\downarrow \downarrow, 10\rangle\right],\right. \\
& \left|\lambda_{2}\right\rangle=\left(4 g^{2}+\Omega_{1}^{2}\right)^{-1 / 2}\left[\left(\Omega_{1}|\uparrow \uparrow, 00\rangle+2 g|\downarrow \uparrow, 01\rangle\right], .\right. \\
& \left|\lambda_{3}\right\rangle=\left(4 g^{2}+\Omega_{2}^{2}\right)^{-1 / 2}\left[\Omega_{2}|\uparrow \downarrow, 00\rangle+2 g|\downarrow \downarrow, 00\rangle\right], \\
& \left|\lambda_{4}\right\rangle=\left(4 g^{2}+\Omega_{2}^{2}\right)^{-1 / 2}\left[\Omega_{2}|\uparrow \uparrow, 00\rangle+2 g|\downarrow \uparrow, 00\rangle\right] .
\end{aligned}
$$

Also we defined the frequencies and parameters by $\Omega_{1}=W-\delta-\Delta^{\prime}, \Omega_{2}=W+\delta-\Delta^{\prime}$. Then using the foregoing eigenvectors we get the wavefunction for any time. For this reason we need to consider the initial state of the quantum dots system. A suitable choice of initial state is a state of Bell. For the sake of generality; we use the initial state of the QDs to be $\left|\psi_{q d}(0)\right\rangle=\left[c_{1}|\uparrow \downarrow\rangle+c_{2} e^{i \phi}|\downarrow \uparrow\rangle\right]$, where $a_{1}$ and $a_{2}$ are real constants satisfying the condition $c_{1}^{2}+c_{2}^{2}=1$.We will consider the initial state of the field to be coherent, or thermal. Then, the initial state for the coupled QD-field system can then be written as

$$
|\Psi(0)\rangle=\left|\psi_{q d}(0)\right\rangle \otimes|00\rangle=\left[c_{1}|\uparrow \downarrow\rangle+c_{2} e^{i \phi}|\downarrow \uparrow\rangle\right] \otimes|00\rangle .
$$

We are now able to find the wavefunction of the system, because the energy eigenstates form a complete set. Using the eigenvalues and eigenvectors (5), together with the initial state (6), (Mitra, Vyas, Erenso, 2007) we obtain the following state vector at the time $t$ :

$$
\begin{aligned}
& |\Psi(t)\rangle=\sum_{k=1}^{N=4} \exp \left(-i \lambda_{E k} t\right)\left\langle\lambda_{E k} \mid \Psi(0)\right\rangle\left|\lambda_{E k}\right\rangle= \\
& e^{-i \lambda_{1} t}\left\langle\lambda_{1} \mid \Psi(0)\right\rangle\left|\lambda_{1}\right\rangle+e^{-i \lambda_{2} t}\left\langle\lambda_{2} \mid \Psi(0)\right\rangle\left|\lambda_{2}\right\rangle+e^{-i \lambda_{3} t}\left\langle\lambda_{3} \mid \Psi(0)\right\rangle\left|\lambda_{3}\right\rangle+e^{-i \lambda_{4} t}\left\langle\lambda_{4} \mid \Psi(0)\right\rangle\left|\lambda_{4}\right\rangle .
\end{aligned}
$$


Due to the orthonormality of the basis vectors we get the coefficients as $x_{i}\left(\Omega_{i}, t\right)=x_{i}(t)=\langle j m, n \mid \Psi(t)\rangle$, and

$$
\begin{aligned}
& x_{1}(t)=c_{1} \frac{\left(\Omega_{1}^{2}+\Omega_{2}^{2}\right) e^{-i\left(\lambda_{1}+\lambda_{3}\right) t}}{\left[\left(4 g^{2}+\Omega_{1}{ }^{2}\right)\left(4 g^{2}+\Omega_{2}{ }^{2}\right)\right]}, \\
& x_{2}(t)=e^{i \phi_{0}}\left[\frac{c_{1} 4 g^{2} e^{-i \lambda_{2} t}}{\left(4 g^{2}+\Omega_{1}{ }^{2}\right)}+\frac{c_{2} 4 g^{2} e^{-i \lambda_{4} t}}{\left(4 g^{2}+\Omega_{2}{ }^{2}\right)}\right], \\
& x_{3}(t)=c_{1} \frac{2 g \Omega_{1}}{\left(4 g^{2}+\Omega_{1}{ }^{2}\right)} e^{-i \lambda_{1} t}, \\
& x_{4}(t)=c_{1} \frac{2 g \Omega_{2}}{\left(4 g^{2}+\Omega_{2}{ }^{2}\right)} e^{-i \lambda_{3} t} .
\end{aligned}
$$

Then the solution of the system in terms of the standard basis can be written as a simple linear combination, i.e.

$$
|\Psi(t)\rangle=x_{1}(t)|\uparrow \downarrow\rangle+x_{2}(t)|\downarrow \uparrow\rangle+x_{3}(t)|\downarrow \downarrow\rangle+x_{4}(t)|\downarrow \downarrow\rangle .
$$

The coefficients $x_{i}(t)$ are given by equations (8). Based on these results that were obtained, in the following section we find the density matrix, as well as reduced density matrix in order to calculate the concurrence and entanglement of formation.

\section{Entanglement of Formation for Two QDs as Qubits Implementation}

For sake of simplicity, let us assume that both cavities are prepared initially in the vacuum state $\left|0_{a}\right\rangle \otimes\left|0_{b}\right\rangle$ and the two QDs are in a pure entangled state specified below as a Bell state. Under these assumptions, there is never more than one photon in each cavity, so the cavity mode is essentially equivalent to a two-level system. This allows a uniform measure of quantum entanglement together to concurrence, for both dots and the cavity modes.

According to the above we must note that there are, in principle, six different concurrences that provide information about the overall entanglements that may arise. We can denote simple form as follows: (Yönac, Yu and Eberly $2006 \& 2007$ ) $C^{A B}$, $C^{A b}, C^{A a}, C^{B b}, C^{A b}, C^{B a}$. Symmetry considerations can provide natural relations among them. Here we confine our attention to $C^{A B}$.

So, it should note that we in reality have six individual systems and four qubits: i.e. the two QDs ( $A$ and $B$, see figure 1) represent two qubits and two cavities ( $a$ and $b$ ) represent other two qubits itself, plus the combinations in interaction between these system as we showed in concurrences. However, we focus only in the $A B$ combination in order to measure the entanglement. For calculate the Entanglement of formation we need find the density matrix in general form of the coefficients $x_{i}(t)$, 
(Yönac, Yu and Eberly 2006 \& 2007) That is, we must to compute the matrix elements, for density reduced matrix, whom work for us in order to find out Spinflipped matrix which is an ingredient essential in Concurrence function for entanglement of formation. Here we show the resulting matrix:

$$
\begin{aligned}
\hat{\rho} & =\left(\begin{array}{cccc}
0 & 0 & 0 & 0 \\
0 & \left|x_{1}\right|^{2} & x_{1} x_{2}^{*} & x_{1}\left(x_{3}^{*}+x_{4}^{*}\right) \\
0 & x_{1}^{*} x_{2} & \left|x_{2}\right|^{2} & x_{2}\left(x_{3}^{*}+x_{4}^{*}\right) \\
0 & x_{1}^{*}\left(x_{3}+x_{4}\right) & x_{2}^{*}\left(x_{3}+x_{4}\right) & \left|x_{3}\right|^{2}+\left|x_{4}\right|^{2}
\end{array}\right), \\
\hat{\rho} & =\left(\begin{array}{cccc}
a & 0 & 0 & w \\
0 & b & z & 0 \\
0 & z^{*} & c & 0 \\
w^{*} & 0 & 0 & d
\end{array}\right) .
\end{aligned}
$$

In the combination of the four qubits that we use as system, appear most the features of character universal. But the simplest is first, all reduced to a two-qubit form, obtained by tracing over the two qubits, will yield a two-qubits mixed state always will have the standard X-form (Yönac M, Yu T and Eberly 2006 and 2007). Where $a+b+c+d=1$. Second, since the concurrence of this mixed state is easily found to be: $C=2 \max \{0,|z|-\sqrt{a d},|w|-\sqrt{b c}\} \equiv 2 \max \{0, Q\}$.

Also, we will encounter the case $w=0$, and this equation turns into: $C=2 \max \{0,|z|-\sqrt{a d}\} \equiv 2 \max \{0, Q\}$. So it is clear that $Q$, defined as

$$
Q=|z|-\sqrt{a d}
$$

This will be an important quantity. Because this have certain conservation properties that derive from $Q$ in some cases because it can be negative, whereas $C$ cannot. The information about the entanglement of two QDs is contained in the reduced density matrix $\rho^{A B}$ for the two dots which can be obtained from expressions (9) and (10) by tracing out the photonic parts of the total pure state. The explicit $4 \times 4$ matrix written in the basis $\{|\uparrow \uparrow\rangle ;|\uparrow \downarrow\rangle ;|\downarrow \uparrow\rangle ; \mid \downarrow \downarrow)\}$ (Yu, Yonac and Eberly 20022007) is given by

$$
\hat{\rho}^{A B}=\left(\begin{array}{cccc}
0 & 0 & 0 & 0 \\
0 & \left|x_{1}\right|^{2} & x_{1} x_{2}^{*} & 0 \\
0 & x_{1}^{*} x_{2} & \left|x_{2}\right|^{2} & 0 \\
0 & 0 & 0 & \left|x_{3}\right|^{2}+\left|x_{4}\right|^{2}
\end{array}\right) .
$$

This is in the standard form of the two-qubit (quantum dots) mixed state, which was noted previously by (Yu T and Eberly J H 2007) in order to two level atoms case. 
Once again the time-dependent matrix elements are given by (5), which we analyzing the case when the detuning is zero: $\Delta^{\prime}=\omega-\varepsilon^{\prime}=\Delta+W=0+W=W$,in resonance. It must be note that only to keep the Förster interaction constant. The total of constants defined into equations for coefficients in equations (8) and eigenvectors (5) are: $\quad \Omega_{1}=W-\delta-\Delta^{\prime}=-2 g, \quad \Omega_{2}=W+\delta-\Delta^{\prime}=2 g \quad$ and $\delta=\left[W\left(W-2 \Delta^{\prime}\right)+\left(4 g^{2}+\left(\Delta^{\prime}\right)^{2}\right)\right]^{1 / 2}=2 g$ therefore the equations (5) and (8) now given by:

$$
\begin{aligned}
& \left|\lambda_{1}\right\rangle=(1 / \sqrt{2})[|\downarrow \downarrow, 10\rangle-|\uparrow \downarrow, 00\rangle], \\
& \left|\lambda_{2}\right\rangle=(1 / \sqrt{2})[|\downarrow \uparrow, 01\rangle-|\uparrow \uparrow, 00\rangle], \\
& \left|\lambda_{3}\right\rangle=(1 / \sqrt{2})[|\uparrow \downarrow, 00\rangle+|\downarrow \downarrow, 00\rangle], \\
& \left|\lambda_{4}\right\rangle=(1 / \sqrt{2})[|\uparrow \uparrow, 00\rangle+|\downarrow \uparrow, 00\rangle] .
\end{aligned}
$$

The coefficients must be normalized. Thus, the constants $c_{1}$ and $c_{2}$ into equations must to obey the normalization condition, also if compared to the eigenvectors obtained in eqs. (13), the latter are entangled states of Bell (resonant case) where the constants are actually of $1 / \sqrt{2}$, except for the sign. Thus the equations for the coefficients are:

$$
\begin{aligned}
x_{1}(t)= & \frac{e^{-i 2 W t}}{8 \sqrt{2} g^{2}} \\
x_{2}(t)= & \frac{e^{i \phi_{0}}}{2 \sqrt{2}}\left[e^{-i(W-g) t}+e^{-i(W+g) t}\right] \\
& =\frac{1}{\sqrt{2}} \cos (g t) e^{-i W t-i \phi_{0}}, \\
x_{3}(t)= & -\frac{1}{2 \sqrt{2}} e^{-i(W-g) t} \\
x_{4}(t)= & \frac{1}{2 \sqrt{2}} e^{-i(W+g) t} .
\end{aligned}
$$

Now we show that the concurrence of the density matrix (12), with references to equation (11), this is given first by function $Q_{A B}(t)$ as:

$$
\begin{aligned}
Q_{A B}(t) & =|z|-\sqrt{a d}=\left|x_{1}^{*} x_{2}+x_{1} x_{2}^{*}\right|-\sqrt{0} \\
& =\frac{1}{16 g^{2}}\left|\cos (g t)\left(e^{-i W t} e^{-i \phi_{0}}+e^{i W t} e^{i \phi_{0}}\right)\right|, \\
Q_{A B}(t) & =\frac{1}{8 g^{2}}\left\{\begin{aligned}
\left|\cos \left(\phi_{0}\right) \cos (g t) \cos (W t)\right| & \Re(\text { part }), \\
\left|\sin \left(\phi_{0}\right) \cos (g t) \sin (W t)\right| & \mathfrak{I} \text { (part). }
\end{aligned}\right.
\end{aligned}
$$

So that the Concurrence function we can be think in dual way, the first one as a function just of time, which is keeping constant, and the another one as a function of two variables, i.e. as function of the time and phase parameter. 
For sake of simplicity we use only the real part in terms of the cosines functions. The imaginary part has a similar behavior. In the figures below we showed several cases for both functions with different values of parameters $W$ and $g$. It must be noted that the plots have a behavior of cosine oscillations type, but self-modulate with a function of the same nature, i.e. cosine-cosine, and the amplitude not exceeding the one, as it should be for Entanglement of Formation. The graphics are showed in next section of results; and the functions for Concurrences are

$$
\begin{aligned}
& C_{A B}(t)=\frac{1}{8 g^{2}}\left|\cos \left(\phi_{0}\right) \cos (g t) \cos (W t)\right|, \\
& \phi_{0}=\text { fixed, } \\
& C_{A B}\left(t, \phi_{0}\right)=\frac{1}{8 g^{2}}\left|\cos \left(\phi_{0}\right) \cos (g t) \cos (W t)\right|, \\
& \phi_{0}=\text { independent variable. }
\end{aligned}
$$

\section{$5 \quad$ Results}

Before presenting graphical results with the plots simulations, first we want to show the analytical results without approach with limit cases on the physical parameters:

$$
\begin{aligned}
& Q_{A B}(t)=\left|\begin{array}{c}
\cos \left(\phi_{0}\right)(\cos (g+W) t+\cos ((g-W) t)) \\
+i \sin \left(\phi_{0}\right)(\sin (g+W) t-\sin ((g-W) t))
\end{array}\right| \times \frac{1}{16 g^{2}}, \\
& Q_{A B}(t)=\frac{1}{8 g^{2}} \cos \left(\phi_{0}\right) \cos (g) t, \quad W=0 .
\end{aligned}
$$

Now, when $g \gg W$, in this case, is the dominant parameter, i.e. the coupling constant between the radiation field and the QDs:

$$
\begin{aligned}
& Q_{A B}(t)=\frac{1}{8 g^{2}}\left|\begin{array}{l}
\cos \left(\phi_{0}\right) \cos W t \cos g t \\
+i \sin \left(\phi_{0}\right) \cos g t \sin W t
\end{array}\right|, \\
& Q_{A B}^{2}(t)=\left(\frac{1}{8 g^{2}}\right)^{2} \cos ^{2} g t\left(\begin{array}{c}
\cos ^{2}\left(\phi_{0}\right) \cos ^{2} W t \\
+\sin ^{2}\left(\phi_{0}\right) \sin ^{2} W t
\end{array}\right), \\
& Q_{A B}(t)=\frac{1}{16 g^{2}}\left|\begin{array}{l}
\cos \left(\phi_{0}\right)(\cos (g+W) t+\cos ((g-W) t)) \\
+i \sin \left(\phi_{0}\right)(\sin (g+W) t-\sin ((g-W) t))
\end{array}\right| .
\end{aligned}
$$

Another interesting case is when we add a parameter $\delta$ to the others parameters, which enables us to get analytical expressions more general, besides being able to manipulate this parameter numerically and perturbative way, i.e., $W=g+\delta ; W \succ \delta, g \succ \delta:$ 


$$
\begin{aligned}
& Q_{A B}(t)=\frac{1}{16 g^{2}}\left|\begin{array}{l}
\cos \left(\phi_{0}\right)(\cos (2 g+\delta) t+\cos (\delta t)) \\
\left.+i \sin \left(\phi_{0}\right)(\sin (2 g+\delta) t+\sin \delta t)\right)
\end{array}\right|, \\
& Q_{A B}^{2}(t)=\left(\frac{1}{16 g^{2}}\right)^{2}\left(\begin{array}{l}
(1+\cos 2 g t)^{2}\left(\begin{array}{l}
\cos ^{2} \delta t \cos ^{2}\left(\phi_{0}\right) \\
+\sin ^{2} \delta t \sin ^{2}\left(\phi_{0}\right)
\end{array}\right) \\
+\sin ^{2} 2 g t\left(\begin{array}{l}
\sin ^{2} \delta t \cos ^{2}\left(\phi_{0}\right) \\
+\cos ^{2} \delta t \sin ^{2}\left(\phi_{0}\right)
\end{array}\right) \\
-2(1+\cos 2 g t) \cos \left(2 \phi_{0}\right) \times \\
\times \sin 2 g t \cos \delta t \sin \delta t
\end{array}\right) .
\end{aligned}
$$

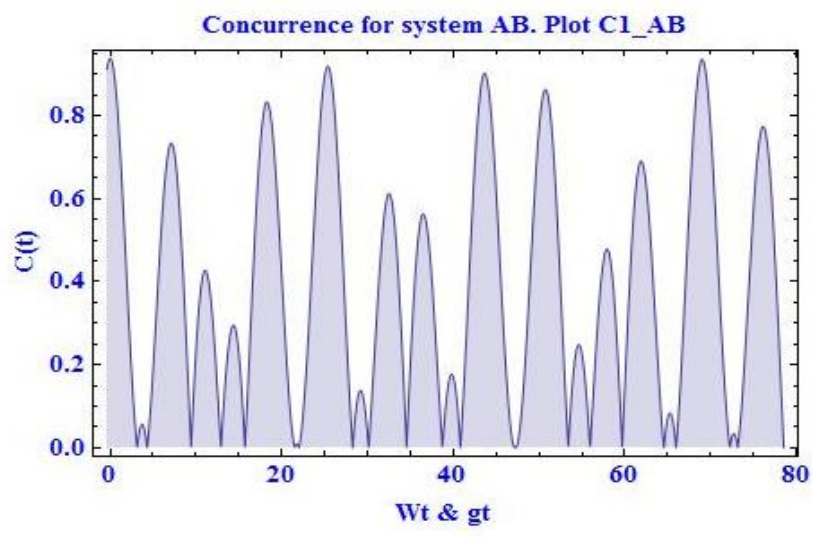

Fig. 2. Plot of the Concurrence for parameters: $W=0.5 ; g=0.365 ; \phi_{0}=0$. In this plot the oscillations fluctuate into of the time interval, almost become of top for entanglement of formation of one.

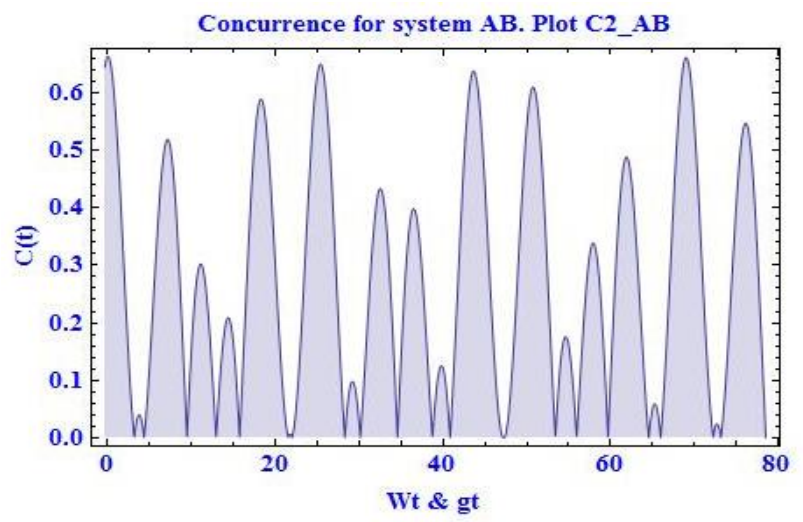

Fig. 3. Plot of the Concurrence for parameters: W=0.5; $g=0.365 ; \phi_{0}=\pi / 4$. In this case the plot decreases the amplitudes of the oscillations, because we do a change of $\pi / 4$ to the phase. 
Now we show the Concurrence plots in order to different parameters values, also in two, and three-dimensionally. The case $3 \mathrm{D}$ we consider the $\phi_{0}$ as variable, which enables us visualize the contour zones of Sudden Death of concurrence.

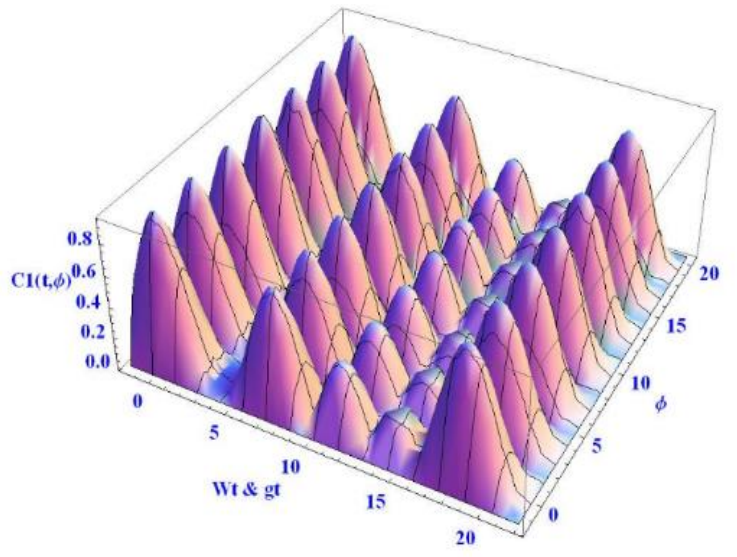

Fig. 4. Plot $3 \mathrm{D}$ for the Concurrence $C_{A B}\left(t, \Phi_{0}\right)$; for parameters: $\mathrm{W}=0.5$; $\mathrm{g}=0.365$.
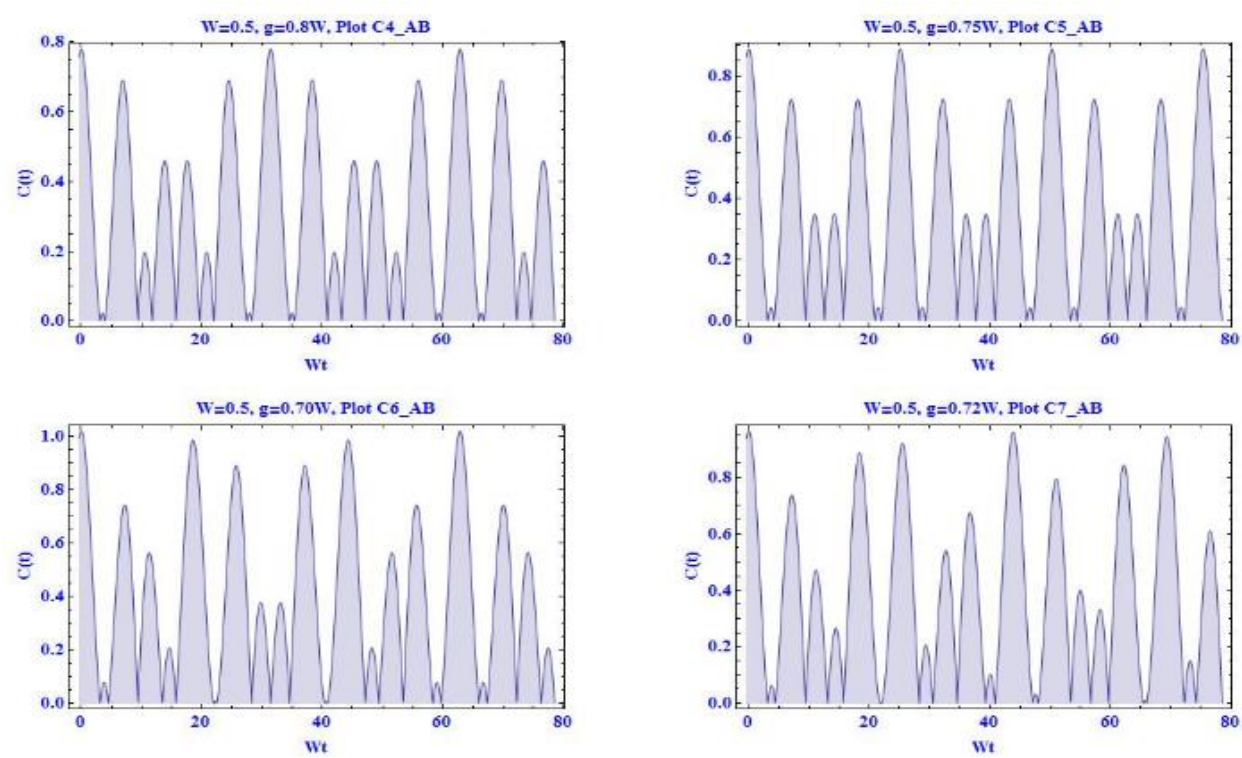

Fig. 5. Concurrence plots $C_{A B}(t)$; for parameters: $\mathrm{W}=0.5$ and plot $4 \mathrm{~g}=0.8 \mathrm{~W}$, plot $5 \mathrm{~g}=0.75 \mathrm{~W}$, Plot6 $\mathrm{g}=0.70 \mathrm{~W}$, and plot7 $\mathrm{g}=0.72 \mathrm{~W}$. We can see that four combinations for constant $\mathrm{g}$ proportional to $\mathrm{W}$. The more optimums combination is in order to plot5, and plot7. Plot6 slightly exceeds the allowable bound for Entanglement of formation and Concurrence of one. This is because the interaction constants differ by a percentage equal (or greater) to $30 \%$, as is clearly noted in the data above. 

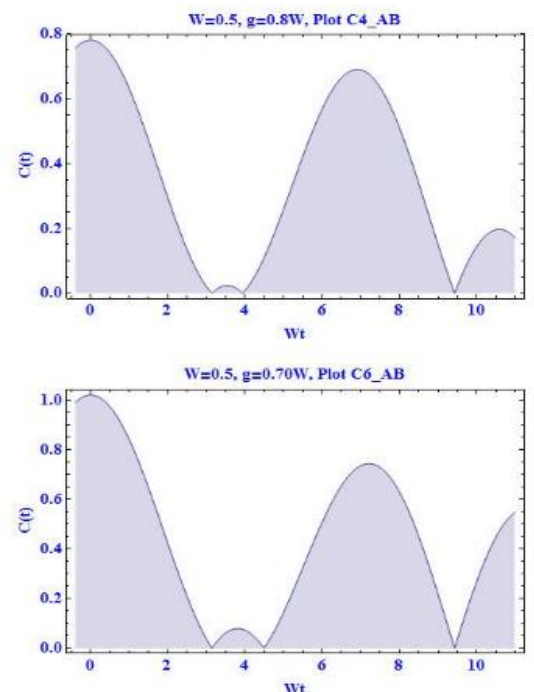
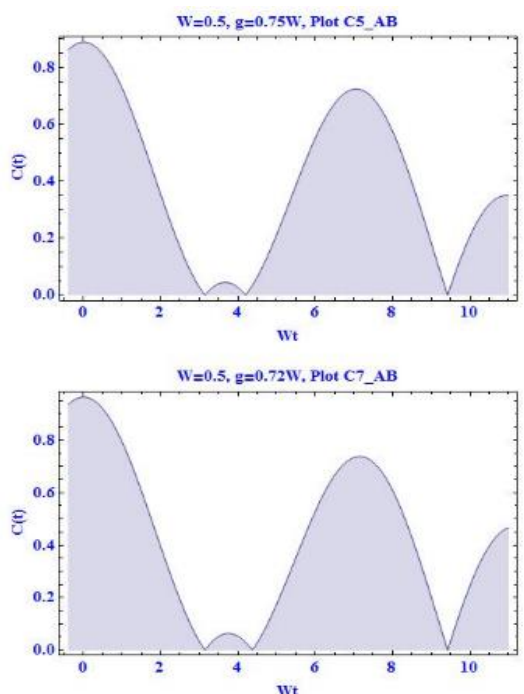

Fig. 6. Concurrence for the same parameters but plotted in a smaller domain, in order to show further details in the zones of minimum of the function $C_{A B}(t)$, the $3 \mathrm{D}$ case with $\left(t, \Phi_{0}\right)$ independent variables is showed in next figure 7 . Parameters: $\mathrm{W}=0.5$ and plot $4 \mathrm{~g}=0.8 \mathrm{~W}$, plot5 $\mathrm{g}=0.75 \mathrm{~W}$, Plot6 $\mathrm{g}=0.70 \mathrm{~W}$, and plot7 $\mathrm{g}=0.72 \mathrm{~W}$.

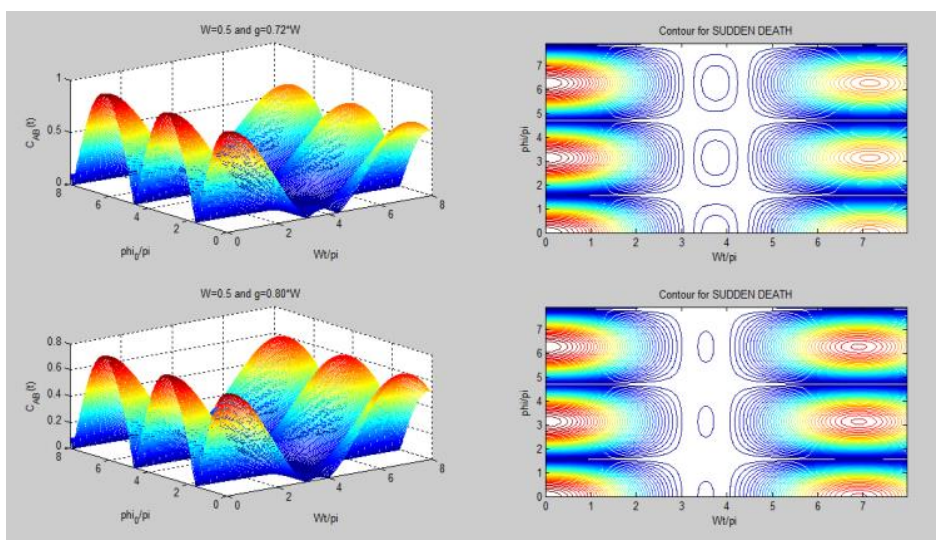

Fig. 7. Plots in $3 \mathrm{D}$ and Contours for so-called SUDDEN DEATH ZONES by $C_{A B}\left(t, \Phi_{0}\right)$; with two cases: $\mathrm{W}=0.5$ and $\mathrm{g}=0.72 \mathrm{~W}, \mathrm{~g}=0.80 \mathrm{~W}$ and the parameter $\Phi_{0}$ is variable, this is consequence of the initial state of Bell. We can see that contour zones of sudden death are minimums of the plots on the right. The interesting is to note that in this small zone rescaled for sake of simplicity that there is not a total sudden death as in the atomic case of the other authors [45-50]. What is also seen in previous plots from figures 2 to 6 into their minimum points. 


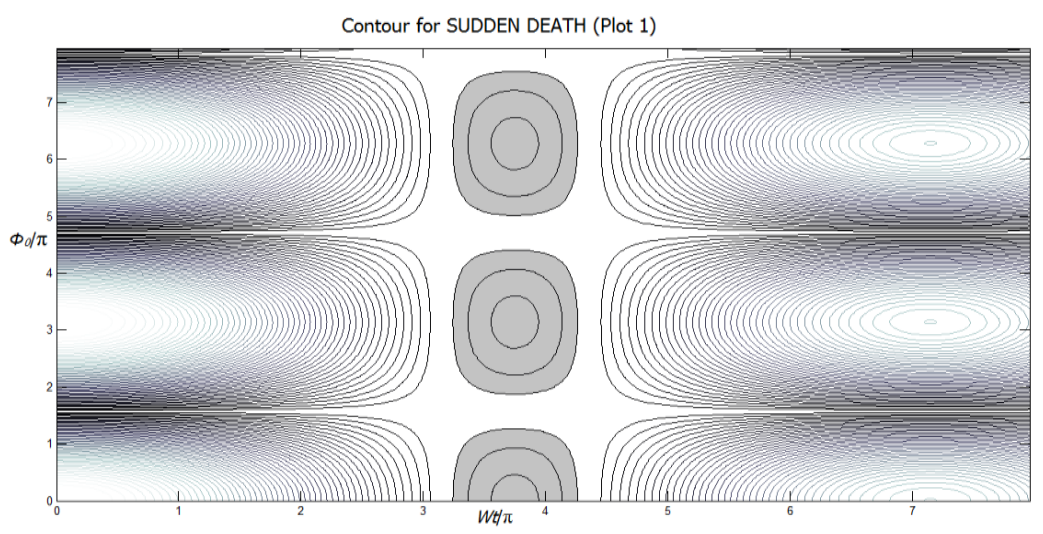

Fig. 8. Plots of Contours for so-called SUDDEN DEATH ZONES by $C_{A B}\left(t, \Phi_{0}\right)$; for the case: $\mathrm{W}=0.5$ and $\mathrm{g}=0.72 \mathrm{~W}$, and the parameter $\Phi_{0}$ is variable, this is consequence of the initial state of Bell. We can see the contour zones for sudden death that are minimums on the plots in gray color, but without become null totally.

\section{Conclusions}

In this paper we study the dynamics behavior of a system of two QDs embedded into own cavity, previously entangled, with initial state type Bell into of the context CQED and Förster interaction included into QDs Hamiltonian. This behavior let us insight the particular dynamics of transference and quantum communication correlations between two qubits, in this case represent for our two QDs, i.e. how is the entanglement process after that qubits are entangled and input into cavity in this situation of communication to distance without interaction, i.e. how evolve this entanglement with the time and consider the initial state of type Bell, which include the $\Phi_{0}$ parameter. The way in order to understand and quantify this process without ambiguity is obtain a secure measure of the entanglement. This measure is the Concurrence and Entanglement of Formation for two qubits (Hill and Wootters 1997; Wootters, 1998) only. The measure is defined only for two qubits as entities of two states, because there is not an extension of this method to more qubits that to allow calculate with precision their entanglement.

Our results between the two cavity-QD, let us see that the entanglement depend of both parameters of interaction, i.e. the interaction field-QDs $(g)$ and the Förster interaction $(W)$. Both interactions must be of the same order, because if either of the two differs significantly from another the result found is that oscillations slightly exceed the bound of the one for entanglement of formation. In this way we find that the interaction parameters must be very well controlled and should not be very different in order of magnitude. Also the best way to control them is by making one of the two in terms of another one by a minimum percentage, as we can see in figures 5 , 6 and 7. Another one very important is the so-called Sudden Death feature where we analyzed in multiples plots in figures 5 and 6 , but with greater accurately in contour plots 7 and 8, how the Sudden Death zones are minimums, however there is not totally 
sudden death of entanglement as happen in the cases study for systems of atoms for other authors (Yu, Yonac and Eberly 2002-2007) or the case for QDs studied in references: (Quiroga-Jhonson 1999; Reina-Quiroga-Jhonson 2000; Mitra, Vyas Erenso 2007), in these papers the authors do not even mentioned the case of Sudden Death as in atomic situation. We can say that for these QDs system almost there is not sudden death because minimum zones are very sharp, i.e. they are smooth curves cosine in which do not we get semi-flat zones, that in atomic case is where entanglement sudden death occurs.

This allows us conclude that our QDs system featuring to two qubits is more efficient for propagation of entanglement without loss of quantum correlation.

Acknowledgment. The author S. Sánchez-Sánchez wants to acknowledge the organization of the international workshop LAOP 2014 held in Cancun, Mexico for the invitation and scholarship for the event. Also, authors S. Sánchez-Sánchez, V. I. Moreno Oliva, and E. Román Hernández acknowledge PROMEP (Programa de mejoramiento para el profesorado de la Secretaria de Educación Pública) by grant support UNISTMO-CA-13 projects in 2013-09 to 2014-09.

\section{References}

1. Bennett, C. H., Brassard, G., Crepeau, C., Jozsa, R., Peres, A., Wootters, W. K.: Phys. Rev. Lett. 70, pp. 1895 (1993)

2. Bennett, C. H., Wiesner, S. J.: Phys. Rev. Lett. 69, pp. 2881 (1992)

3. Bennett, C. H., DiVincenzo, D. P., Smolin, J. A., Wootters, W. K.: Phys. Rev. A 54 pp. 3824 (1996)

4. Bennett, C. H., Bernstein, H. J., Popescu, S., Schumacher, B.: Phys. Rev. A 53, pp. 2046 (1996)

5. Bennett, C. H., Brassard, G., Popescu, S., Schumacher, B., Smolin, J., Wootters, W. K.: Phys.Rev. Lett. 76, pp. 722 (1996)

6. DiVincenzo, D. P.: Science. 270, pp. 255 (1995)

7. Deutsch, D.: Quantum theory, the Church-Turing principle and the universal quantum computer. In: Proc. R. Soc., London, A 400, pp. 97-117 (1985)

8. Deutsch, D: Quantum computational networks. In: Proc. R. Soc., London, A 439, pp. 553-558 (1989)

9. Feynman, R. P.: Quantum mechanical computers. Optics News, 11, pp. 11-20 (1985)

10. Fuchs, C. A., Gisin, N., Griffiths, R. B., Niu, C. S., Peres, A.: Phys. Rev. A 56, pp. 1163 (1997)

11. Hill, S., Wootters, W. K.: Phys. Rev. Lett. 78, pp. 5022 (1997)

12. Joos, E, Zeh, H. D, Kiefer, C, Giulini, D, Kupsch, K., Stamatescu, I. O.: Decoherence and the Appearance of a Classical World in Quantum Theory. Germany: Springer (2003)

13. Kane, B. E., Loss, D., DiVincenzo, D. P.: Phys. Rev. A 57, pp. 120 (1998)

14. Burkard, D., Loss, D., DiVincenzo, D. P.: Phys. Rev. B 59, pp. 2070 (1999)

15. Barenco, A., Deutsch, D., Ekert, A., Jozsa, R.: Phys. Rev. Lett. 74, pp. 4083 (1995)

16. Imamoglu, A., Awschalom, D. D., Burkard, G., DiVincenzo, D. P., Loss, D., Sherwin, M., Small, A., Mitra, A., Vyas, R., Erenso, D., Phys. Rev. A 76 (2007)

17. Nielsen, M. A., Chuang, I. L.: Quantum Computation and Quantum Information. CambridgeUniversity Press, Cambridge, England (2000)

18. Quiroga, L., Johnson, N. F.: Phys. Rev. Lett. 83, pp. 2270 (1999)

19. Reina, J. H., Quiroga, L., Johnson, N. F.: Phys. Rev. A. 62 (2000) 
20. Sanchez-Sanchez, S.: Dynamic behavior of collective and reduced Quantum dots system. Instituto Nacional de Astrofísica Óptica y Electrónica (INAOE), PhDr. Thesis, Tonantzintla, Puebla, Mexico (2011)

21. Sanchez-Mondragon, J. J., Alejo-Molina, A., Sanchez-Sanchez, S., Torres-Cisneros, M.: Comparison of the Dicke Model and the Hamiltonianfor n Quantum Dots, Quantum Dots, Nanoparticles, and Nanoclusters II. In: Proceedings of SPIE, Vol. 5734 (2005)

22. Alejo-Molina, A., Sánchez-Mondragón, J. J., Sánchez-Sánchez, S.: Detuning Colectivo Del Modelo De L Puntos Cuánticos. XLVIII Congreso Nacional Smf / Xviii Reunion Anual Amo Guadalajara Jalisco (2005)

23. Sánchez-Sánchez, S., Sánchez-Mondragón, J. J., Castillo-Soria, F. R.: Representación de Puntos Cuánticos en la Base Atómica Coherente. Memorias en extenso del LII Congreso Nacional de Física (SMF)/ Reunión Anual de Óptica (AMO), Acapulco Guerrero (2009)

24. Werner, R. F.: Phys. Rev. A 40, pp. 4277 (1989)

25. Peres, A.: Phys. Rev. Lett. 77, pp. 1413 (1996)

26. Wootters, W. K.: Phys. Rev. Lett. 80, pp. 2245 (1998Yönac M, Yu T and Eberly J H, J. Phys. B: At. Mol. Opt. Phys. 39, S621-S625 (2006)

27. Yönac, M., Yu, T., Eberly, J H.: J. Phys. B: At. Mol. Opt. Phys. 40, pp. S45-S59 (2007)

28. Yu, T., Eberly, J. H.: Phys. Rev. Lett. 93 (2004)

29. Yu, T., Eberly, J. H.: Opt. Commum. at press Preprint quant-ph/0602196 (2006)

30. Yu, T., Eberly, J. H.: 2002 Phys. Rev. B 66 193306;

31. Yu, T., Eberly, J. H.: Quantum Inf. Comp. 7 in press, Preprint quant-ph/0503089 (2007) 